\title{
Bullying en estudiantes de Secundaria de las Instituciones educativas públicas de la Provincia de Huaura.2016
}

Bullying in secondary students of the public educational institutions of the Province of Huaura.2016

\begin{abstract}
Maritza Isabel Martínez Loli', Norma Adela Moreno Rivera1', María Rosario Meza Aguirre², Marcelo Gumercindo Zúñiga Rojas ${ }^{1}$, Perfecta Zobeida Fernández Guzmán ${ }^{3}$, Fátima del Rocio Bernal Regalado', Bethsabeth Clara
\end{abstract} Melosich Aviles ${ }^{1}$, Luz Mery Tadeo Sifuentes ${ }^{1}$, Salazar Vega Olga Xuxa ${ }^{1}$

\section{RESUMEN}

Objetivo: Identificar las prácticas de bullying en los estudiantes de secundaria de las Instituciones Educativas Públicas de la Provincia de Huaura. 2016. Métodos: EEs una investigación básica de nivel descriptivo, diseño no experimental corte transversal. La población está formada por 1660 estudiantes de nivel secundario de 12 instituciones educativas estatales de la Provincia de Huaura. La muestra de tipo no probabilístico porque esta direccionada a alumnos del $1^{\circ}$ año de nivel secundario entre 11 y 15 años y una muestra de 586 estudiantes que equivale a $35 \%$ del total. Para la recolección de datos se empleó el Cuestionario de Bullying a estudiantes elaborado por el equipo de investigadores, validado a través de método de extracción teniendo como resultado 0,950 y una confiabilidad a través del alfa de cronbach de 0,819 que corresponde a alta confiabilidad; está comprende cuatro dimensiones del bullying: físico, psicológico, verbal y social y consta de 18 ítems y 3 alternativas (siempre, a veces y nunca). Para el procesamiento de la información se utilizó el programa estadístico SPSS versión 20. Resultados: Las prácticas de bullying se encuentran presentes en los estudiantes de secundaria de las Instituciones Educativas Públicas de la Provincia de Huaura, durante el año académico 2016 . El bullying físico es de $61,1 \%$ el psicológico en $79,9 \%$, el verbal se halló un $72,6 \%$ y finalmente el social un 10,2\%. Existen diferencias de percepción del bullying en cuanto al tipo de familia de donde proceden, encontrándose mayores porcentajes en los estudiantes de hogares nucleares y menores porcentajes en estudiantes de hogares monoparentales, extensos y combinados. Existen diferencias de percepción de bullying en relación a género, las alumnas refieren en un $27,7 \%$ en contraste con un $41,1 \%$ de los alumnos. Conclusion: Existe prácticas de bullying en sus diversas formas en los estudiantes de secundaria de las Instituciones Educativas Públicas de la Provincia de Huaura. 2016, siendo el de mayor porcentaje el psicológico y verbal.

Palabras clave: Bullying, estudiantes secundarios, autoestima. 


\title{
Bullying en estudiantes de Secundaria de las Instituciones educativas públicas de la Provincia de Huaura.2016
}

Bullying in secondary students of the public educational institutions of the Province of Huaura.2016

Maritza Isabel Martínez Loli', Norma Adela Moreno Rivera', María Rosario Meza Aguirre², Marcelo Gumercindo

Zúñiga Rojas ${ }^{1}$, Perfecta Zobeida Fernández Guzmán ${ }^{3}$, Fátima del Rocio Bernal Regalado', Bethsabeth Clara Melosich Aviles ${ }^{1}$, Luz Mery Tadeo Sifuentes ${ }^{1}$, Salazar Vega Olga Xuxa ${ }^{1}$

\begin{abstract}
Objective: Mldentify bullying practices in high school students of the Public Educational Institutions of the Province of Huaura, 2014. Methods: It is a basic investigation of descriptive level, nonexperimental design cross section. The population is formed by 1660 students of secondary level of 12 state educational institutions of the Province of Huaura. The sample of non-probabilistic type because it is addressed to students of the 1st year of secondary level between 11 and 15 years. The sample of non-probabilistic type because it is addressed to students of the 1 st year of secondary level between 11 and 15 years and a sample of 586 students that is equivalent to $35 \%$ of the total. For the data collection, the Student Bullying Questionnaire prepared by the research team was used, validated through the extraction method, resulting in 0.950 and reliability through the cronbach alpha of 0.819 , which corresponds to high reliability; it comprises four dimensions of bullying: physical, psychological, verbal and social and consists of 18 items and 3 alternatives (always, sometimes and never). For the processing of the information the statistical program SPSS version 20 was used. Results: The bullying practices are present in the secondary students of the Public Educational Institutions of the Province of Huaura, during the 2016 academic year. Physical bullying is $61.1 \%$ psychological bullying in $79.9 \%$, verbal bullying was $72.6 \%$ and finally social bullying $10.2 \%$. There are differences in the perception of bullying in terms of the type of family from which they come, with higher percentages in students from nuclear homes and lower percentages in students from single-parent, extended and combined households. There are differences in perception of bullying in relation to gender, the students refer in $27.7 \%$ in contrast to $41.1 \%$ of the students. Conclusions: There are bullying practices in its various forms in high school students of the Public Educational Institutions of the Province of Huaura, 2016. the psychological and verbal being the highest percentage.
\end{abstract}

Keywords: Bullying, secondary students. 


\section{INTRODUCCIÓN}

El bullying se ha convertido en una problemática social-educativa que afecta a estudiantes de los niveles de educación primaria y educación secundaria, por ello es importante subrayar que la vida de los estudiantes implica la práctica de relaciones interpersonales satisfactorias para garantizar una adecuada convivencia; entendiendo que las relaciones interpersonales comprenden toda la variedad de capacidades que un estudiante debe adquirir para desenvolverse en forma eficiente, con asertividad, con respeto hacia sus compañeros, ante las diferencias, críticas, diversidad de culturas, razas, rasgos físicos entre otros. Las relaciones interpersonales adecuadas les van a permitir una mejor convivencia, trabajo en equipo, por ende una permanencia agradable dentro de la institución educativa. Sin embargo cuando en la interacción social no se sabe respetar o se empieza a burlar de los demás a través de insultos, de risas burlescas o utilizar otras formas inadecuadas de hacer "sentir mal" la otra persona estamos hablando ya de un maltrato conocido como bullying.

El Bullying nombre en inglés hace referencia a todo tipo de maltrato que sucede dentro del contexto educativo, este término fue utilizado por Olweus, (1972) cuando detecta casos de maltrato escolar con consecuencias de tres suicidios. En el Bullying se agrupan el acoso escolar, el hostigamiento, la manipulación social, la coacción, la exclusión social, la intimidación, llegando estos tipos de maltrato incluso a través de las redes sociales lo que agrava más la situación porque la víctima no solo desarrolla miedo a permanecer dentro de la Institución Educativa sino también a hacer uso de las redes sociales porque siente que más personas se dan cuenta de su papel de víctima.

Al respecto Olweus (1998) citado en Avilés (2005) refiere que un alumno es agredido o se convierte en victima cuando está expuesto de forma repetida y durante un tiempo a acciones que lleva a cabo un alumno o varios de ellos. Cabe resaltar que el bullying tiene factores condicionantes y una trayectoria así como también consecuencias, es probable que un estudiante que ha participado de bullying en la primaria en cualquiera de los roles, continúe esa práctica en los estudios secundarios, de esto se tiene referencia en investigaciones realizadas en diferentes instituciones educativas tanto a nivel nacional como internacional.

A esta situación se le debe agregar el componente familiar. Es necesario señalar la importancia de la familia en la formación de la personalidad es un elemento condicionante porque la familia es el primer agente socializador del niño y las investigaciones afirman que los modelos de familia pueden ser predictores de conductas violentas en los niños. Las familias con niveles adecuados de comportamiento, comunicación y que satisfacen las necesidades básicas de sus integrantes respetándose entre ellos y con reglas claras de convivencia, formarán niños capaces de respetar y valorar a los demás (Cueva, 2012). En tanto que los niños provenientes de hogares conflictivos y con necesidades afectivas, por lo general tenderán a desarrollar conductas inadecuadas de relación con los demás, es así que creemos abordar desde la tipología familiar.

Por otro lado, es necesario conocer la presencia del Bullying en relación a la identidad de género en la población de estudio, muchas investigaciones han señalado que el tipo de intimidación interfiere con la forma en que construyen la identidad de género los y las estudiantes y las diferencias en el tipo de relaciones que se dan entre mujeres y mujeres, hombres y hombres y mujeres y hombres y que dichas diferencias y que tanto están conectadas con la presencia del bullying que se desarrollan las alumnas y los alumnos de secundaria. Entre las consecuencias más importantes que genera este fenómeno particular se pueden encontrar, su incidencia en el bajo rendimiento, en la deserción escolar y casos de suicidio que se han reportado incidiendo negativamente en las relaciones interpersonales.

Para tal efecto se ha revisado investigaciones a 
nivel internacional y nacional, tales como: Cueva, (2012). La familia como detonante del fenómeno Bullying Universidad Central de Ecuador. Tuvo como objetivo conocer la relación entre la funcionalidad familiar y el fenómeno Bullying. El problema identificado de acoso escolar en altas cantidades, motiva a probar que, a menor nivel de funcionalidad familiar mayor grado de victimización dentro del fenómeno Bullying. Se fundamenta en la teoría ecológica de Bronfenbrenner, que destaca la importancia de los ambientes de la interacción social y la influencia de ésta en el desarrollo conductual; tratado en dos capítulos: Bullying y Familia. Investigación correlacional, no experimental, con método inductivo deductivo y estadístico. A una muestra de ciento ochenta y cuatro niños/as entre nueve a once años, estudiantes escolares, se ha evaluado acoso escolar y disfuncionalidad familiar con cuestionarios adaptados y estructurados, los resultados evidencian disfuncionalidad familiar, que correlacionados con datos del fenómeno Bullying se establecen porcentajes altos de niños acosadores con características vulnerables ante este fenómeno. Se recomienda la presencia de un profesional Psicólogo/a en la institución para un mejor manejo de los estudiantes.

Yandiria, (2011). Relación del bullying con comportamientos discriminatorios por cuestiones de género en la construcción de la identidad de género. Universidad La Salle. Bogotá. Tuvo por objetivo determinar la relación que existe entre el bullying y los comportamientos discriminatorios por género en el proceso de construcción de la identidad de género en los estudiantes de secundaria. Investigación exploratorio, mixto, que emplea elementos de la metodología cualitativa, de la cual toma el diseño de la Teoría Fundada para el análisis de las entrevistas en profundidad y la cuantitativa, con el fin de identificar patrones generales en la población a través del instrumento psicométrico INSEBULL (Instrumento para la medición del bullying). Les permitió identificar casos de agresión entre iguales en un grupo de estudiantes de secundaria, además de la identificación de un tipo de intimidación con contenido sexual o bullying sexual y la influencia que existe entre los comportamientos de bullying por cuestiones de género en la construcción de la identidad de género de los estudiantes, lo que señala que las agresiones que se dan al interior de las clases son basadas en definiciones reduccionistas sobre lo que es ser hombre y mujer, caracterizadas por el uso de chistes machistas, comentarios sexistas y discriminatorios, los cuales interfieren con la percepción que tiene de sí mismos, pues las víctimas empiezan a ser identificadas por las causas de sus agresiones, por lo que terminan aceptando y creyendo dichas agresiones, además de aceptar la cultura de los agresores con el fin de permanecer en el sistema social del curso.

CCoicca, T, (2010). Bullying y funcionamiento familiar en una Institución Educativa del Distrito de Comas. Universidad Nacional Federico Villarreal Se analiza la relación entre el bullying y funcionamiento familiar, en una muestra de 261 escolares del nivel secundario (131 mujeres y 130 varones), del Distrito de Comas. La investigación asume un diseño no experimental, de tipo descriptivo-correlacional. Los instrumentos utilizados fueron el Auto-test Cisneros y el Apgar familiar. El nivel de bullying acumulado es de $58,3 \%$. El bullying hallado según género es de $56 \%$ para varones y el $64,3 \%$ para mujeres. La modalidad más frecuente es poner apodos $20,3 \%$.Los niveles de funcionamiento familiar evidencian que el $32.5 \%$ presenta una buena función familiar, el $42,9 \%$ una disfunción leve, el 16,4\% una disfunción moderada y el $8 \%$ una disfunción grave. También se halló que existe una correlación negativa débil entre bullying y el funcionamiento familiar (Rho $-0,198 p<0,01$ ). La correlación por género entre las dos variables reportó un índice de (Rho $-0,221 p<$ 0,011 ) para mujeres y (Rho $-0,161 p<0,068$ ) para varones, evidenciando la existencia de una correlación negativa débil. Y la correlación por el lugar de origen de los escolares de la muestra reportó una correlación positiva débil de (Rho 0,189 con una $p<0,002$ ).

En esta investigación se pretende identificar las diferentes prácticas de bullying en las instituciones educativas en las dimensiones: física, psicológica, verbal y social; todo esto nos permita señalar y diferenciar las razones que expliquen científicamente que la violencia es un 
comportamiento de los individuos, pero el bullying se ha convertido en un problema educativo psicosocial y legal que afecta a la convivencia escolar, Cerezo (2006).

En tal sentido, la preocupación del equipo de investigadores es establecer la existencia de los niveles de prácticas Bullying en el ámbito escolar de la Provincia de Huaura, sus dimensiones establecidas, tipo de familia e identidad de género.

Las prácticas de bullying se encuentran presenten en los estudiantes de secundaria de las Instituciones Educativas Públicas de la Provincia de Huaura, 2016.

Siendo el objetivo identificar las prácticas de bullying en los estudiantes de secundaria de las Instituciones Educativas Públicas de la Provincia de Huaura, 2016.

\section{MATERIAL Y MÉTODOS}

Es una investigación básica de nivel descriptivo, cuyo objetivo es recolectar información relevante en una muestra respecto a un mismo fenómeno, variables o aspecto de interés y luego caracterizar este fenómeno, diseño no experimental de corte transversal. La población está formada por 1660 estudiantes de 18 instituciones educativas estatales del nivel secundario de la Provincia de Huaura. La muestra de tipo no probabilístico porque esta direccionada a alumnos del 1 er año de nivel secundario que ascendió a 586 estudiantes que equivale a $35 \%$ del total. Para la recolección de datos se empleó el Cuestionario de Bullying a estudiantes elaborado por el equipo de investigadores, validado a través de juicio de expertos y método de extracción teniendo como resultado 0,950 y una confiabilidad a través del alfa de cronbach de 0,819 que corresponde a alta confiabilidad; está comprende cuatro dimensiones del bullying: físico, psicológico, verbal y social y consta de 18 ítems y 3 alternativas (siempre, a veces y nunca) que se detalla en la tabla 1. Para el procesamiento de la información se utilizó el programa estadístico SPSS versión 20.
Tabla 1. Distribución de dimensiones, indicadores e ítems del cuestionario de Bullying a estudiantes

\begin{tabular}{|c|c|c|}
\hline $\begin{array}{c}\text { Dimensiones } \\
\text { de Bullying } \\
\end{array}$ & Indicadores & Ítems \\
\hline Física & $\begin{array}{l}\text { Golpes, empujones, } \\
\text { patadas }\end{array}$ & $\begin{array}{l}7,8,9,14,15 \\
16,17\end{array}$ \\
\hline Psicológica & $\begin{array}{l}\text { Burlas, satisfacción } \\
\text { destructiva }\end{array}$ & $2,3,6,18$ \\
\hline Verbal & $\begin{array}{l}\text { Insultos, groserías, } \\
\text { apodos }\end{array}$ & $\begin{array}{l}1,4,5,10,11 \\
17\end{array}$ \\
\hline Social & Aislamiento & 12 \\
\hline
\end{tabular}

\section{RESULTADOS}

Para conocer la confiabilidad del Cuestionario de Bullying a estudiantes se aplicó el estadístico de Alfa de Cronbach, obteniendo 0,819 .

Tabla 2. Estadístico de fiabilidad de Cuestionario de Bullying a estudiantes

\begin{tabular}{ccc}
\hline $\begin{array}{c}\text { Alfa de } \\
\text { Cronbach }\end{array}$ & $\begin{array}{c}\text { Alfa de Cronbach } \\
\text { basada en los } \\
\text { elementos } \\
\text { tipificados }\end{array}$ & N de elementos \\
\hline, 819 &, 821 & 18 \\
\hline
\end{tabular}

Con respecto a la edad de los estudiantes, (Tabla 3 ) se encontró que la edad promedio es de 12 años, la edad mediana que parte en dos la información de 12, la edad modal que se repite es 12 y las edades oscilan entre 11 años y 15 años de edad.

Así mismo, de acuerdo a los señalados por la Organización Mundial de la salud tenemos que el $9,4 \%$ se encuentran en 11 años señalados como pre adolescencias, de 12 a 14 años de edad está referido a la etapa de la adolescencia temprana en un $88,8 \%$ y solo el $1,7 \%$ en la adolescencia tardía.

Tabla 3. Distribución de frecuencias y porcentajes de la edad de la población de estudio.

\begin{tabular}{ccccc}
\hline Edad & Frecuencia & Porcentaje & $\begin{array}{c}\text { Porcentaje } \\
\text { válido }\end{array}$ & $\begin{array}{c}\text { Porcentaje } \\
\text { acumulado }\end{array}$ \\
\hline 11 & 55 & 9,4 & 9,4 & 9,4 \\
12 & 346 & 59 & 59 & 68,4 \\
13 & 139 & 23,7 & 23,7 & 92,2 \\
14 & 36 & 6,1 & 6,1 & 98,3 \\
15 & 10 & 1,7 & 1,7 & 100 \\
\hline Total & 586 & 100 & 100 & \\
\hline
\end{tabular}


Por otro lado, el mayor porcentaje se encuentra en la edad de 12 años con un $59 \%$ del total de la población, tal como señala la Figura 1.

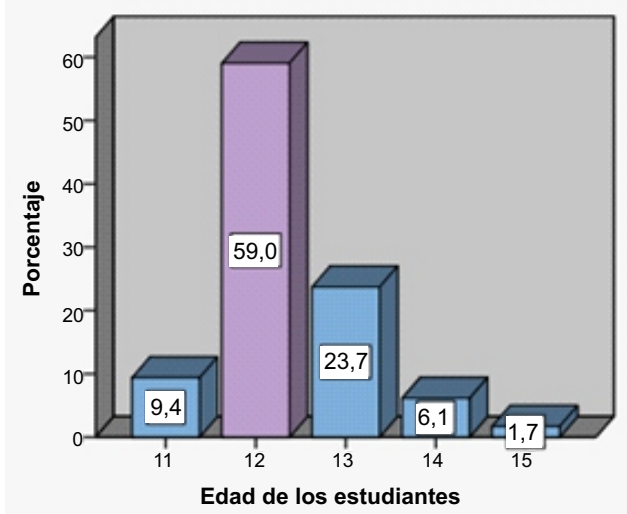

Figura 1. Distribución de porcentajes de las edades de la población de estudio

Con respecto al género tenemos que el $49 \%$ son de sexo femenino y el $51 \%$ son de sexo masculino, demostrando la predominancia de varones en la muestra. Figura 2.

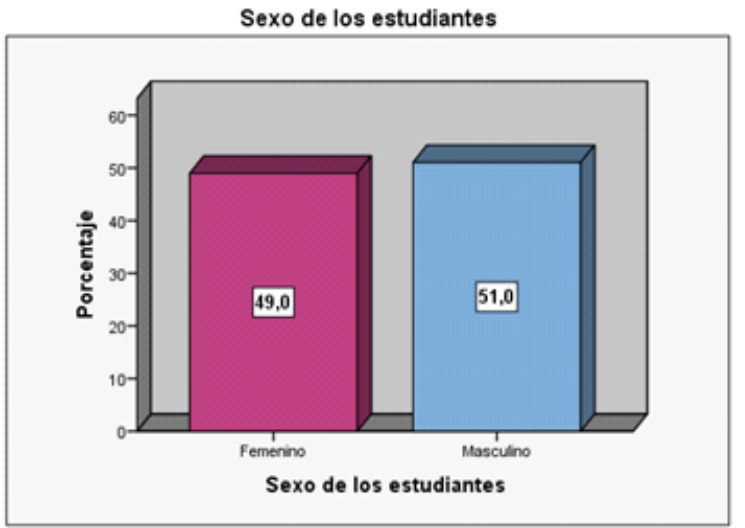

Figura 2. Distribución de porcentajes según género
En la Figura 3, referente al tipo de familia de la población de estudio, se puede observar que el 52,6 viven den familias nucleares con padres y hermanos, el $23,5 \%$ proceden de familias combinadas, padres, hermanos, abuelos y otros familiares, el $20,1 \%$ señalaron que viven con familias monoparentales es decir con padre o madre y hermanos y por último el $3,8 \%$ afirmaron que proceden de familias extensas.

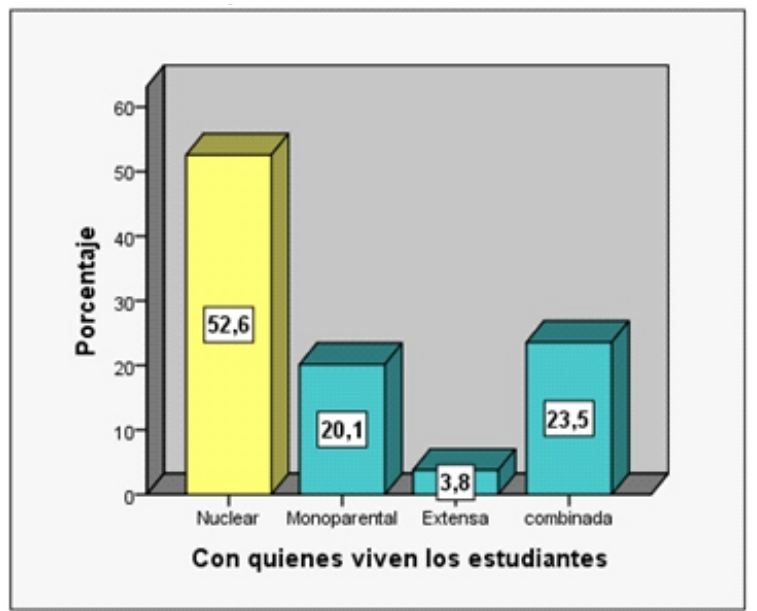

Figura 3. Distribución de porcentajes según tipo de familia

En relación a las instituciones educativas tenemos que se aplicaron el cuestionario a 18 colegios de los distritos de Huacho (4), Huaura (3), Santa María (2), Hualmay (2), Vègueta (3), Carquin (1) Sayán (2) y Paccho (1) la mayoría de estudiantes el $11,1 \%$ pertenecen a la I.E. Generalísimo Don José de San Martin- Huaura y existe menor porcentaje de 2,2\% respectivamente de la Institución educativa de Paccho. Figura 3.
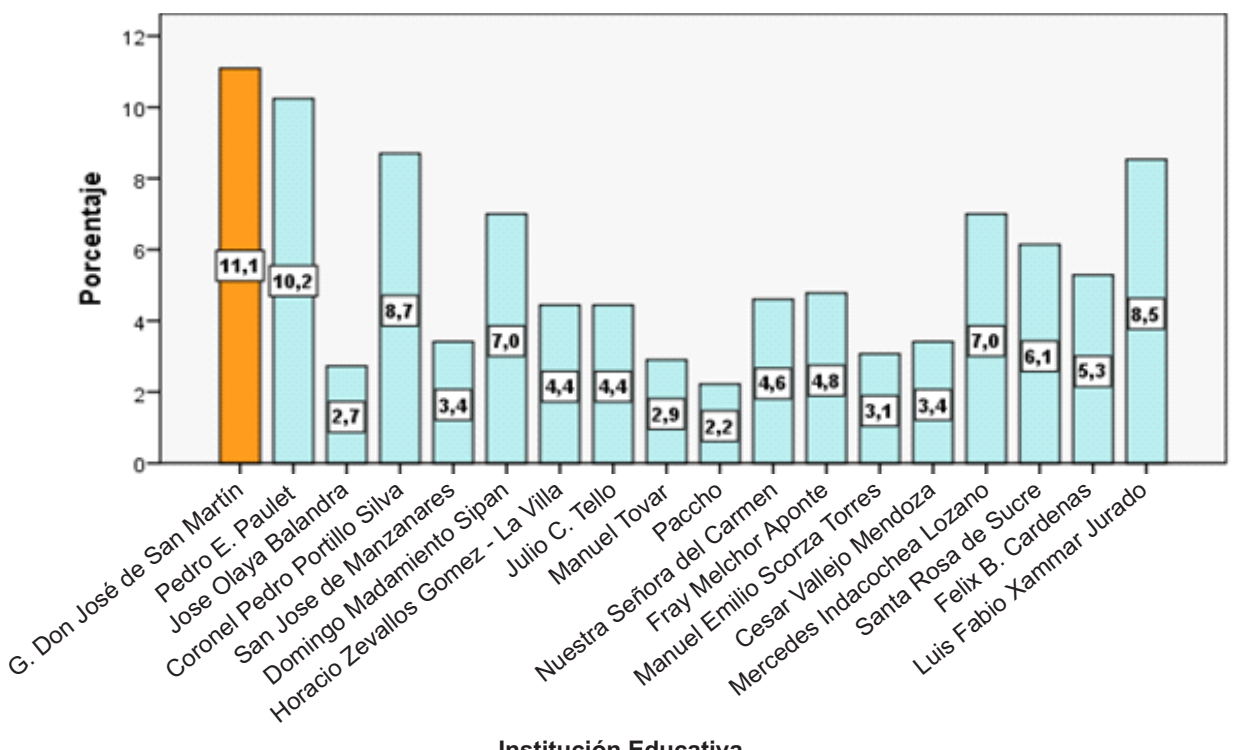

Figura 3. Distribución de porcentajes de las instituciones educativas estatales encuestadas 
La Tabla 4, demuestra que la práctica del bullying se encuentra presente en los estudiantes del primer año de secundaria de las instituciones educativas de secundaria en la Provincia de Huaura. El 48,8\% refieren los estudiantes que a veces se da esta práctica, el $31,2 \%$ dice que nunca se da y el $20 \%$ manifiesta que no hay bullying, sin embargo si se suman los porcentajes de a veces y siempre se tiene que un $68,8 \%$ si práctica o es víctima de bullying, por lo tanto queda demostrado la hipótesis general.

Tabla 4. Distribución de frecuencias y porcentajes de prácticas de Bullying en la población de estudio.

\begin{tabular}{cccc}
\hline & & Frecuencia & Porcentaje \\
\hline \multirow{4}{*}{ Válidos } & Nunca & 183 & 31,2 \\
& A veces & 286 & 48,8 \\
& Siempre & 117 & 20,0 \\
& Total & 586 & 100,0 \\
\hline
\end{tabular}

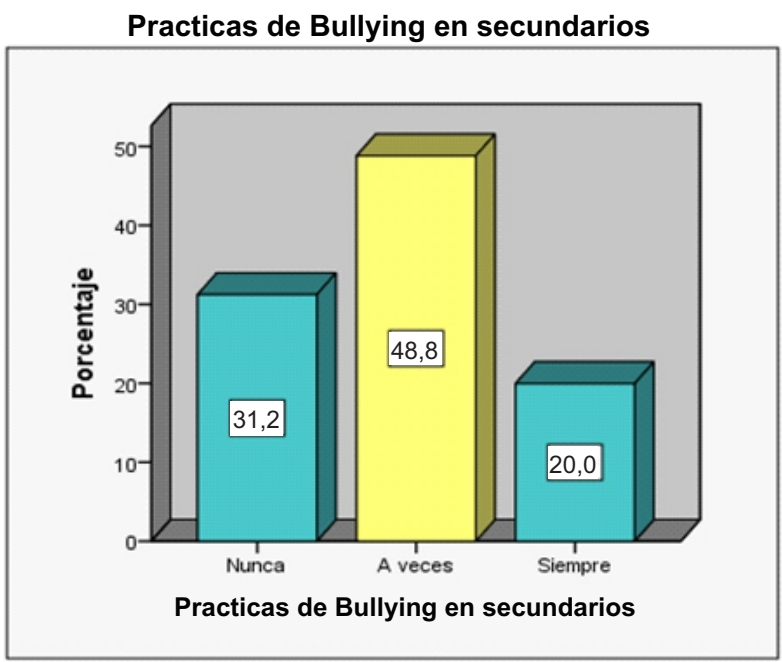

Figura 4. Distribución porcentual de las prácticas de Bullying en secundarios

Se encontró en las alumnas mujeres que un $22,9 \%$ percibe que a veces hay bullying, un $4,8 \%$ dice que siempre se da el bullying que sumados los porcentaje tenemos que 27,7 refiere la existencia de bullying; mientras que el $25,9 \%$ de los alumnos varones manifiestan que a veces se presenta el bullying y un $15,2 \%$ refiere que siempre sumando tenemos que un $41,1 \%$ acepta que hay bullying. Encontrándose diferencias significativa de percepción de bullying según género. (Tabla 5).

Tabla 5. Distribución de frecuencias y porcentajes de prácticas de Bullying y género.

\begin{tabular}{cccccc}
\hline \multirow{2}{*}{ Sexo de los estudiantes } & \multicolumn{3}{c}{$\begin{array}{c}\text { Prácticas de Bullying en } \\
\text { secundarios } \\
\text { A }\end{array}$} & Nunca & Total \\
& & veces & Siempre & \\
\hline \multirow{2}{*}{ Femenino } & Recuento & 125 & 134 & 28 & 287 \\
& \% del total & $21,3 \%$ & $22,9 \%$ & $4,8 \%$ & $49,0 \%$ \\
Masculino & Recuento & 58 & 152 & 89 & 299 \\
& \% del total & $9,9 \%$ & $25,9 \%$ & $15,2 \%$ & $51,0 \%$ \\
Total & Recuento & 183 & 286 & 117 & 586 \\
& \% del total & $31,2 \%$ & $48,8 \%$ & $20,0 \%$ & $100,0 \%$ \\
\hline
\end{tabular}

Para una mejor presentación se visualiza la Figura 5, donde se observa las frecuencias según género, entendiendo que mayoritariamente los estudiantes varones manifiestan a veces y siempre existe la presencia de practica de bullying en el centro educativo a diferencia de las estudiantes mujeres que afirman que nunca y a veces existe la presencia de practica de bullying en el centro educativo, por lo que queda demostrada la hipótesis general encontrándose diferencias significativa de percepción de bullying según género.

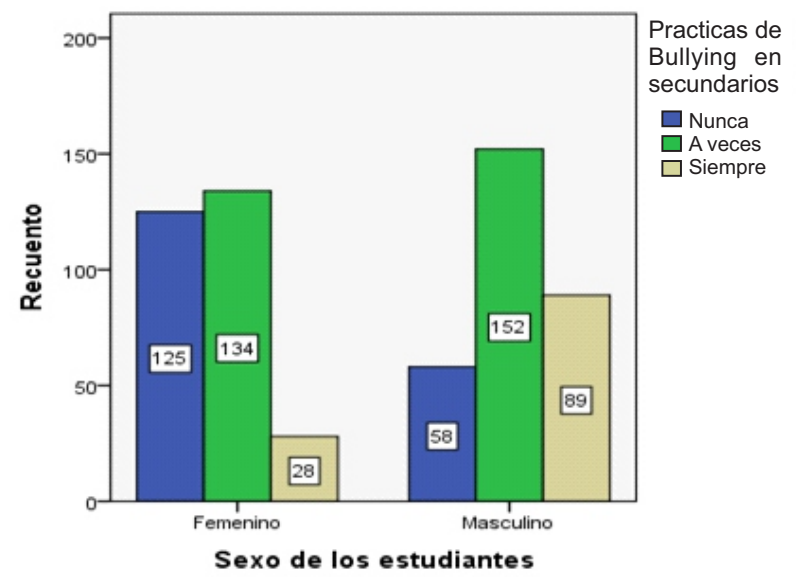

Figura 5. Distribución de frecuencias de las prácticas de bullying según género

En la Figura 6, al analizar cada forma de bullying tenemos la práctica de bullying físico es de $61,1 \%$ (a veces $32,8 \%$ y siempre $28,3 \%$ ), en el bullying psicológico el $79,9 \%$ practica el bullying (a veces $64,7 \%$ y siempre $15,5 \%$ ) en el bullying verbal se halló un $72,6 \%$ que practican el bullying (a veces $55,5 \%$, y siempre 17,1 ) y finalmente en el bullying social se encuentra en $10,2 \%$ (a veces $8,5 \%$ y siempre $1,7 \%$ ). 


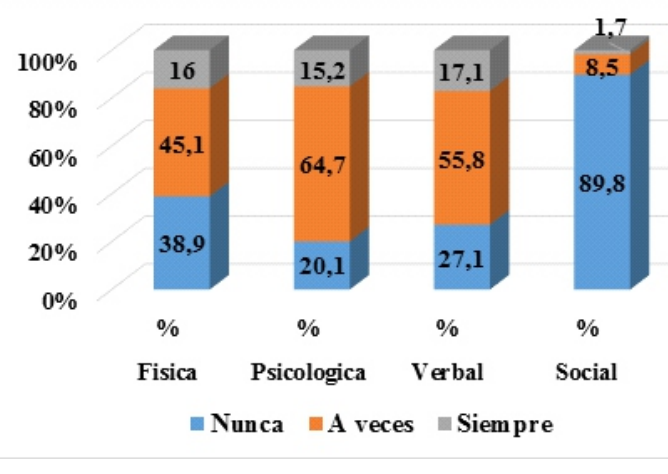

Asimismo, en la Tabla 7 se muestra los estadísticos descriptivos, donde se observa las diferencias de medias en las formas de prácticas de bullying, donde la media más elevada se encuentra en el bullying psicológico y el menos elevado en el bullying social. Por otro lado se observa diferencias en la desviación típica de las diferentes formas de prácticas que perciben los estudiantes, por lo que queda demostrado la hipótesis especifica 1.

Figura 6. Distribución de porcentajes de formas de práctica de bullying

Tabla 7. Estadísticos de formas de Bullying en secundarios

\begin{tabular}{cccccc}
\hline & & Físico & Psicológico & Verbal & Social \\
\hline \multirow{2}{*}{$\mathrm{N}$} & Válidos & 586 & 586 & 586 & 586 \\
\cline { 2 - 4 } & Perdidos & 0 & 0 & 0 & 0 \\
Media & 1,77 & 1,95 & 1,90 & 1,12 \\
Mediana & 2,00 & 2,00 & 2,00 & 1,00 \\
Moda & 2 & 2 & 2 & 1 \\
Desv. típ. &, 706 &, 593 &, 658 & 1 \\
Mínimo & 1 & 1 & 1 & 3 \\
\hline
\end{tabular}

En la Tabla 8, al analizar tipo de familia y prácticas de bullying, tenemos que el $34,7 \%$ señala prácticas de bullying (a veces $25,8 \%$ y siempre $8,9 \%$ ) y proceden de familias nucleares de padres y hermanos, así mismo; el $15 \%(9,7 \%$ a veces y $5,3 \%$ siempre $)$ afirman que existen prácticas de bullying y proceden de familias son monoparentales miembros que viven con un solo padre o madre y hermanos y por último el $16,7 \%$ ( 1,4 a veces y $5,3 \%$ siempre) manifiestan que existe prácticas de bullying y proceden de familias combinadas cuyos miembros familiares son padres, hermanos, abuelos y otros parientes.

Tabla 8. Distribución de frecuencias y porcentajes de prácticas de bullying y tipo de familia

\begin{tabular}{ccccc} 
& \multicolumn{2}{c}{ Prácticas de Bullying en secundarios } & \\
\cline { 2 - 3 } & Nunca & A veces & Siempre & Total \\
Nuclear & 105 & 151 & 52 & 308 \\
& $17,9 \%$ & $25,8 \%$ & $8,9 \%$ & $52,6 \%$ \\
Monoparental & 30 & 57 & 31 & 118 \\
& $5,1 \%$ & $9,7 \%$ & $5,3 \%$ & $20,1 \%$ \\
Extensa & 8 & 11 & 3 & 22 \\
& $1,4 \%$ & $1,9 \%$ & $0,5 \%$ & $3,8 \%$ \\
combinada & 40 & 67 & 31 & 138 \\
& $6,8 \%$ & $11,4 \%$ & $5,3 \%$ & $23,5 \%$ \\
Total & 183 & 286 & 117 & 586 \\
& $31,2 \%$ & $48,8 \%$ & $20,0 \%$ & $100,0 \%$ \\
\hline
\end{tabular}


En la Figura 7 , se puede observar las diferencias en cuanto a percepción que tienen los estudiantes sobre las prácticas de bullying y la procedencia de su hogares, donde predomina dicha percepción en alumnos que proceden de hogares nucleares, quedando demostrada la hipótesis.

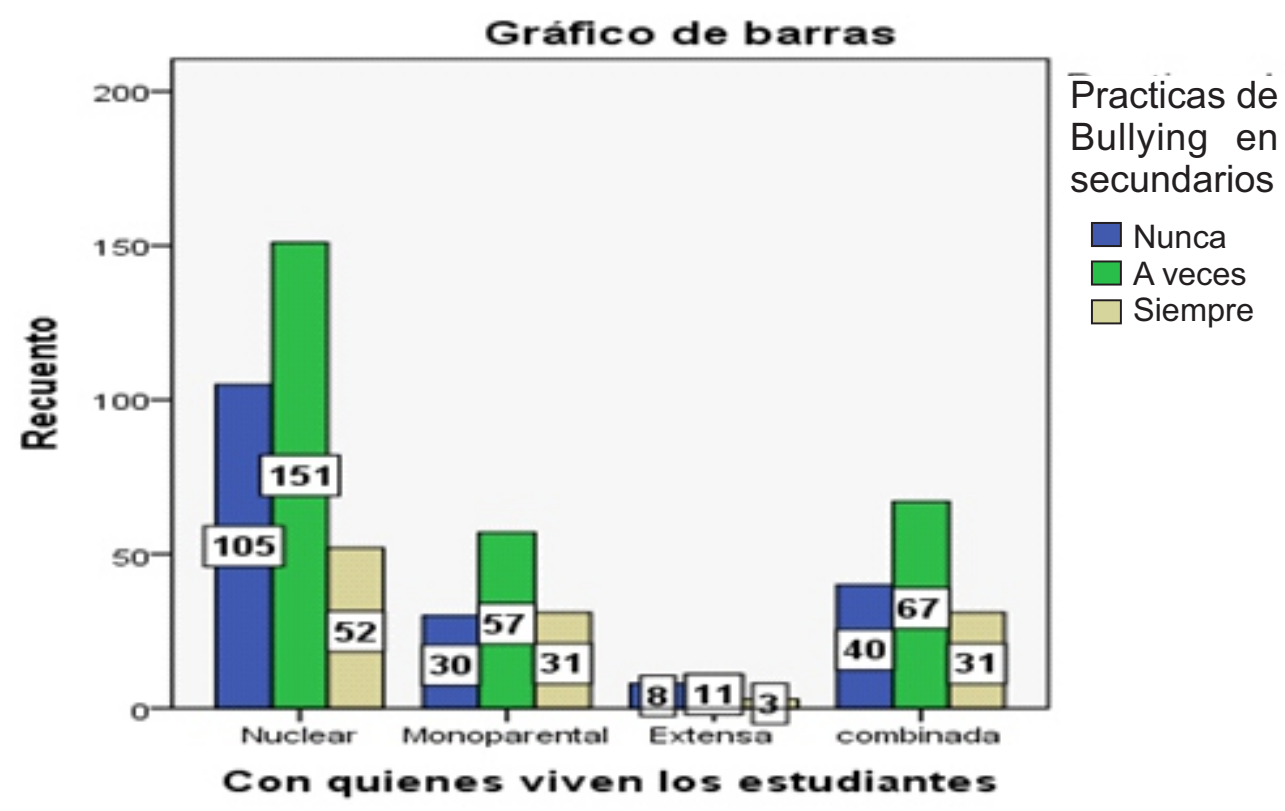

Figura 7. Distribución de frecuencias de prácticas de bullying y tipo de familia

En la Figura 7, se puede observar las diferencias en cuanto a percepción que tienen los estudiantes sobre las prácticas de bullying y la procedencia de su hogares, donde predomina dicha percepción en alumnos que proceden de hogar

\section{DISCUSIÓN}

Olwens (1998) citado en Aviles (2005) resalta que el bullying tiene factores condicionantes y una trayectoria así como también consecuencias, es probable que un estudiante que ha participado de bullying en la primaria en cualquiera de los roles, continúe esa práctica en los estudios secundarios. Y en esta investigación con estudiantes de secundaria se ha demostrado que las prácticas de bullying se encuentran presente en los estudiantes de secundaria de las Instituciones Educativas Públicas de la Provincia de Huaura. 2016 en sus diversas formas cuyos resultados son como sigue: bullying físico $61,1 \%$ (a veces $32,8 \%$ y siempre $28,3 \%$ ), bullying psicológico $79,9 \%$ (a veces $64,7 \%$ y siempre $15,5 \%$ ), bullying verbal $72,6 \%$ (a veces $55,5 \%$, y siempre 17,1 ) y bullying social $10,2 \%$ (a veces $8,5 \%$ y siempre
$1,7 \%)$; y haciendo una deducción lógica, diremos que la práctica de bullying en estos estudiantes se desarrolló desde la primaria.

Cueva (2012), afirma que "los modelos de familia pueden ser predictores de conductas violentas en los niños. Las familias con niveles adecuados de comportamiento, comunicación y que satisfacen las necesidades básicas de sus integrantes respetándose entre ellos y con reglas claras de convivencia, formarán niños capaces de respetar y valorar a los demás". Se entiende por capacidad de respetar y valorar cuando no nos burlamos, no insultamos y no discriminamos. En esta investigación al analizar tipo de familia y prácticas de bullying, tenemos que el $34,7 \%$ señala prácticas de bullying (a veces $25,8 \%$ y siempre $8,9 \%$ ) y proceden de familias nucleares de padres y hermanos, así mismo; el 15\% (9,7\% a veces y $5,3 \%$ siempre) afirman que existen prácticas de bullying y proceden de familias son monoparentales miembros que viven con un solo padre o madre, el 16,7\% (1,4 a veces y $5,3 \%$ siempre) manifiestan que existe prácticas de bullying y proceden de familias combinadas cuyos miembros familiares son padres, 
hermanos, abuelos y otros parientes y un 2,8\% proceden de familias extensas. Con estos resultados se contradice lo vertido por Cueva.

Yandiria, (2011) "identificó los casos de agresión entre iguales en un grupo de estudiantes de secundaria, además encontró un tipo de intimidación con contenido sexual o bullying sexual y la influencia que existe entre los comportamientos de bullying por cuestiones de género en la construcción de la identidad de género de los estudiantes, lo que señaló que las agresiones que se daban al interior de las clases eran basadas en definiciones reduccionistas sobre lo que es ser hombre y mujer, caracterizadas por el uso de chistes machistas, comentarios sexistas y discriminatorios, los cuales interferían con la percepción que tiene de sí mismos, pues las víctimas empezaron a ser identificadas por causas de sus agresiones, por lo que terminaba aceptando y creyendo dichas agresiones, además de aceptar la cultura de los agresores con el fin de permanecer en el sistema social del curso". Estos resultados demuestran que un $22,9 \%$ de las alumnas perciben que a veces hay bullying, un $4,8 \%$ dice que siempre se da el bullying que sumados los porcentaje tenemos que $27,7 \%$ refiere la existencia de bullying; mientras que el $25,9 \%$ de los alumnos varones manifiestan que a veces se presenta el bullying y un $15,2 \%$ refiere que siempre sumando tenemos que un $41,1 \%$ acepta que hay bullying. Encontrándose diferencias significativas de percepción de bullying según género, sin embargo el bullying se da en ambos géneros.

CCoicca, T, (2010) encontró que "el bullying según género es de $56 \%$ para varones y el $64,3 \%$ para mujeres. En estos resultados encontramos discrepancia con la investigación de CCoicca, T (2010), mientras que su investigación demostró mayor incidencia de bullying en mujeres, nosotros hemos demostrado que el mayor porcentaje se da en varones. Además el mismo autor ha demostrado "en los niveles de funcionamiento familiar que el $32,5 \%$ presenta una buena función familiar, el $42,9 \%$ una disfunción leve, el $16,4 \%$ una disfunción moderada y el $8 \%$ una disfunción grave". En la investigación se ha demostrado que los estudiantes que proceden de hogares nucleares perciben la presencia de bullying en mayor porcentaje que aquellos que proceden de otros tipos de hogares como los monoparentales, extendidos o combinados.
Se puede observar las diferencias en cuanto a percepción que tienen los estudiantes sobre las prácticas de bullying y la procedencia de sus hogares, donde predomina dicha percepción en alumnos que proceden de hogares nucleares.

Existen diferencias de percepción de bullying en relación a género, se ha demostrado que las alumnas refieren la existencia de bullying en un $27,7 \%$ en contraste con un $41,1 \%$ de los alumnos. Encontrándose diferencias significativas de percepción de bullying según género, sin embargo en las instituciones educativas de la Provincia de Huaura se da el bullying en ambos géneros.

Se concluyó que las prácticas de bullying se encuentran presenten en los estudiantes de secundaria de las Instituciones Educativas Públicas de la Provincia de Huaura. 2016.

\section{Agradecimientos}

Al Director de la Institución Educativa Estatal Pedro E. Paulet quien avaló la realización de la investigación. A los directores, docentes y estudiantes de las Instituciones Educativas Estatales que accedieron a la aplicación de los instrumentos de recolección de datos y que permitió arribar a los resultados antes mencionados

\section{REFERENCIAS BIBLIOGRÁFICAS}

Aviles, Jose María (2005). EL maltrato entre escolares en el contexto de las conductas de acoso. Bullying en la escuela. Modelo de intervención. Es paña. Recuperado en: $\mathrm{http} / / \mathrm{www}$.elrefugio.net/b

CCoicca, T, (2010). Bullying y funcionamiento familiar en una Institución Educativa del Distrito de Comas. Tesis para optar el título profesional de Licenciado en Psicología. Universidad Nacional Federico Villarreal, Lima, Perú.

Cerezo, F. (2006). Violencia y victimización entre escolares. El bullying: estrategias de identificación y elementos para la intervención a través del Test Bull-S. Electronic journal of research in educational psychology, 4(9), 333-352. 
Cueva, M. (2012). La familia como detonante del fenómeno Bullying. Informe final del Trabajo de Titulación de Psicóloga Clínica. Carrera de Psicología Clínica. Universidad Central Del Ecuador, Quito.

Olweus, D. (1972). Personality and aggression. In Nebraska Symposium on Motivation. Nebraska Symposium on Motivation, Vol. 20, 261).

Yandiria, Y. (2011). Relación del bullying con comportamientos discriminatorios por cuestiones de género en la construcción de la identidad de género. Proyecto de investigación para optar para al título de Licenciada en inglés, francés y español, Universidad de La Salle, Bogotá.
Correo electrónico:

martinez@unjfsc.edu.pe

Revisión de pares:

Recibido: 15-05-2017

Aceptado: 26-06-2017 